\title{
Contention Resolution in OBS Using FDL_WA_WPremp
}

\author{
Suriani Mohd Sam, Hamdan Sayuti, Norsheila Fisal, and Norliza Mohamed
}

\begin{abstract}
As optical burst switched networks offer connectionless transport, there exists the chance that burst may contend with one another at intermediate nodes. Contention will happen if multiple bursts from various input ports are meant for the same output port all at once. we introduce a new approach FDL_WA_WPremp to reduce the burst loss probability during contention. through simulation and analytical modeling, it is shown that the policy introduced reduces burst loss substantially when compared to the standard policy of dropping the contending burst in the event of contention.
\end{abstract}

Index Terms-OBS, contention resolution, FDL, wavelength assignment, preemption, burst loss.

\section{INTRODUCTION}

Optical burst switching was introduced for optical WDM networks. Circuit switching employs two-way reservation method that has a large round trip. Packet switching alternatively, has a large buffer requirement, complex control and strict synchronization issues. OBS is designed to achieve a balance between the coarse-grained circuit switching and fine-grained packet switching. As such, a burst may be considered as having an intermediate granularity as compared to circuit and packet switching.

Packets delivered at an OBS ingress node that are meant for the same egress OBS node and belong to the similar QoS class are combined and sent in bursts. At intermediate nodes, the data within the optical signal is transparently switched to the subsequent node according to forwarding information contained inside a control packet preceding the burst. At the egress node, the burst is subsequently deassembled and sent out electronically.

Given that optical burst switched networks is using connectionless transport, there exists the likelihood that bursts might contend with one another at intermediate nodes. Contention will happen if multiple bursts from different input ports are intended for the same output port at the same time. Contention resolution schemes may be used to provide QoS in an all-optical core network.

Since OBS networks use connectionless transport, the bursts will have the possibility to contend with one another at the intermediate nodes. Contention happens when more than one burst attempt to reserve the same wavelength

Manuscript received February 9, 2014; revised May 16, 2014.

Suriani Mohd Sam is with UTMSPACE, University Teknology Malaysia, Jalan Semarak, Kuala Lumpur, Malaysia (e-mail: suriani@ic.utm.my)

Hamdan Sayuti and Norsheila Fisal are with Electrical Engineering Faculty, Universiti Teknologi Malaysia, Skudai, Johor, Malaysia (e-mail: hamdan@utm.my,Sheila@fke.utm.my)

Norliza Mohamed is with Razak School, Electrical Engineering Departement, Universiti Teknologi, Malaysia (e-mail: mnorliza@ic.utm.my) channel on an outgoing network. Burst loss that is caused from contention is a key issue in OBS networks. Such contention losses which are temporary in nature can lower the performance at the upper layers.

In electronic network, contention is resolved by buffering the contending bursts. In spite of this, optical buffers are complicated to apply and there is no optical equivalent of random access memory. In OBS network, when contention occur one of the contending data burst is allowed to reserve the channel, for other data bursts one or a combination of the contention resolution techniques can be applied. If contention still unable to resolved, therefore one of the contending data burst is dropped. There are four main ways of resolving contention in OBS. The first two are the use of fiber delay lines (FDL) to delay the burst for a discrete and finite period [1]-[3] and deflecting the burst to another output fiber, known as deflection routing [4]-[7]. The third resolution is by implementing wavelength conversion [8] and [9] where a contending burst is sent on another wavelength through wavelength conversion. The forth is the burst segmentation [10]-[13] which a contending burst is broken into segments and the overlapped part of the contending burst is dropped.

The paper is organized as follows. In Section II, the proposed QoS enhancement in OBS is briefly discussed. In Section III, the analytical approach used in the simulation is presented. The propose network model and the simulation environment is presented in Section IV meanwhile Section $\mathrm{V}$ discusses on the simulation results and its performance. The conclusion of this paper is presented in Section VI.

\section{Proposed QoS Enhancement IN OBS}

The main technology restrictions on optical edge and core switching are the lack of optical memories and the limitations of electronic/optical conversion devices. Therefore, in this work, Fiber Delay Line (FDL) is proposed to delay the burst at the edge nodes when no wavelengths are available during the limited time period. To further reduce the burst loss probability, the FDL technique is integrated with wavelength assignment and preemption schemes that are being deployed at the core nodes to achieve better performance. The propose scheme is known as FDL_WA_WPremp, which the performance will be analyzed simultaneously into the network model proposed.

\section{A. Fiber Delay Line (FDL) at The Ingress Router}

In connectionless transport environment, competition among the burst in the OBS networks occurs at the intermediate nodes. Contention happens if more than one burst attempt to reserve the same wavelength channel on an outgoing link. In other words, burst loss due to contention is a main issue of interest in OBS networks. Although these 
losses are momentary, but it can degrade the performance at the higher layers. Although optical buffering implementing fiber delay lines (FDLs) to delay the transmission of bursts is considered to be the most successful technique, but it comes with some drawbacks that is with extra delay and additional expenditure of the FDLs.

In this propose work, the FDL is employed between the edge router and the core router; i.e. between IR1, IR2 to CR1 and IR3, IR4 to CR2 as illustrated in Fig. 1. The FDL approach that is implemented in this work has the reference value of the study proposed in [14]. In spite of having a certain length $D$ to delay the light, the propose work has converted it in terms of delaying the data bursts by introducing variable delay time to the link between each edge node to core node so that data burst will have less probability of reaching the core at the same time. The study stated that 133.869 meters of minimal FDL length can be use in order to obtain a reasonable or optimum blocking rate for a link of $10 \mathrm{Gbps}$. Since in the propose work, the link between IR and CR is set to be $2.5 \mathrm{Gbps}$, therefore the FDL in terms of delaying the burst on the link with respect to time is about $540 \mathrm{~ns}$. Table III, Table V summarize the delay time between each OBS edge node and its corresponding core nodes that are used throughout the simulation works to study the burst loss probability.

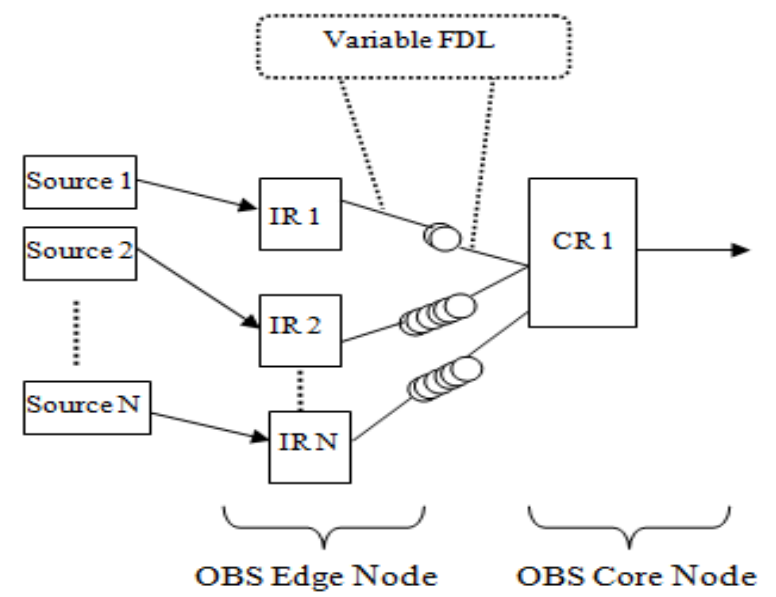

Fig. 1. Proposed FDL approach.

\section{B. Wavelength Assignment and Preemption (WA_WPremp) at the Core Router}

Developments of worthy and resourceful wavelength assignment policies are essential. Such policies are extremely important in OBS networks than in wavelength routed optical networks, owing to the fact that in the former, a burst is sent out without initially reserving resources along the path. Consequently, a burst may be discarded at any intermediate switch down its path, even as it enters its last hop prior to the destination, resulting in considerable waste of network resources.

Besides the significant outcome of the wavelength assignment yield, another effective solution to contention resolution technique is the wavelength preemption scheme in providing relative service differentiation. In relative service differentiation, the QoS of one class is classified moderately to other classes. Preemption is the process where high priority traffic has the dominant role to take over the low priority traffic wavelength in this approach of wavelength preemption.

By integrating these two substantial techniques, the burst loss probability will definitely be minimized when comparing it with its individual performance in the OBS network. Referring to the proposed core node architecture in Fig. 2, the traffic classes of EF, AF and BE are assigned each with a predetermined wavelength of $10 \mathrm{Gbps}$ capacity. $\mathrm{EF}$ is assigned to Lambda 1, Lambda 2 is allocated for AF traffic, whereas BE is being served by Lambda 3 .

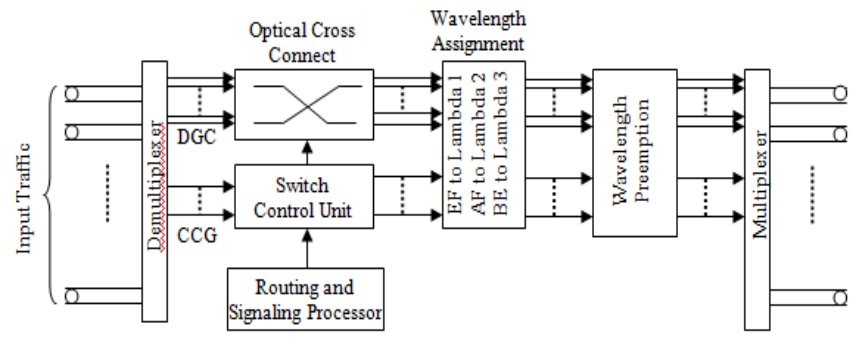

Fig. 2. Proposed core node architecture.

In this scheme, there is a table for each wavelength to update its bandwidth utilization from time to time as the bursts travel through it. The bandwidth of each link is capped at only $90 \%$ of its capacity. When it reaches the capped value, then wavelength preemption technique will take into action and that depends on the traffic priority. In this allocation, Lambda 3 is the lowest priority wavelength. When the allocation bandwidth of Lambda 1 is fully utilized by the EF traffic, the remaining EF burst that need to be sent out will not be dropped but the wavelength of Lambda 3 will be preempted to carry the EF traffic and the consequences BE burst will be dropped. Furthermore, if it comes to worst case scenario, if Lambda 3 is still insufficient to carry the EF traffic, then Lambda 2 will be preempted. In case of the AF traffic, it has the authority to preempt Lambda 3 whereas as for BE traffic, no preemption activity is allowed and therefore, its data bursts are discarded from the network system.

\section{ANALYTICAL APPROACH}

The analytical approach that will be used in this work is the burst blocking probability (BLP) specifically for the performance measure of optical burst switching network. Blocking in a DiffServ Optical Burst Switching network can be modeled with the $\mathrm{M} / \mathrm{G} / \mathrm{k} / \mathrm{k}$ queue or mostly known as Erlang B where bursts are not allowed to wait. In this system, $k$ represents the number of wavelengths used at each output port. Evaluation in done based on three classes of traffic. For this analysis, we assume that the basic offset time of each class is the same, which means that the basic offset time does not affect class isolation. The overall burst blocking probability in multi-classes OBS node can be obtained by using the aforementioned Erlang B formula [15].

$$
P_{b}=\frac{\left(\frac{\lambda}{\mu}\right) k / k !}{\sum_{i=0}^{k} \frac{\left(\frac{\lambda}{\mu}\right)^{k}}{i !}}
$$


where; $P_{b}$ is the burst blocking probability (BLP)

$\lambda$ is the connection arrival rate

$\mu$ is the service rate

The evaluation of the burst loss probability will be focused on implementation of the propose QoS enhancement in OBS particularly using FDL, FDL_WA and FDL_WA_WPremp with different offered traffic being increased and a different burst assembly schemes employed in the network.

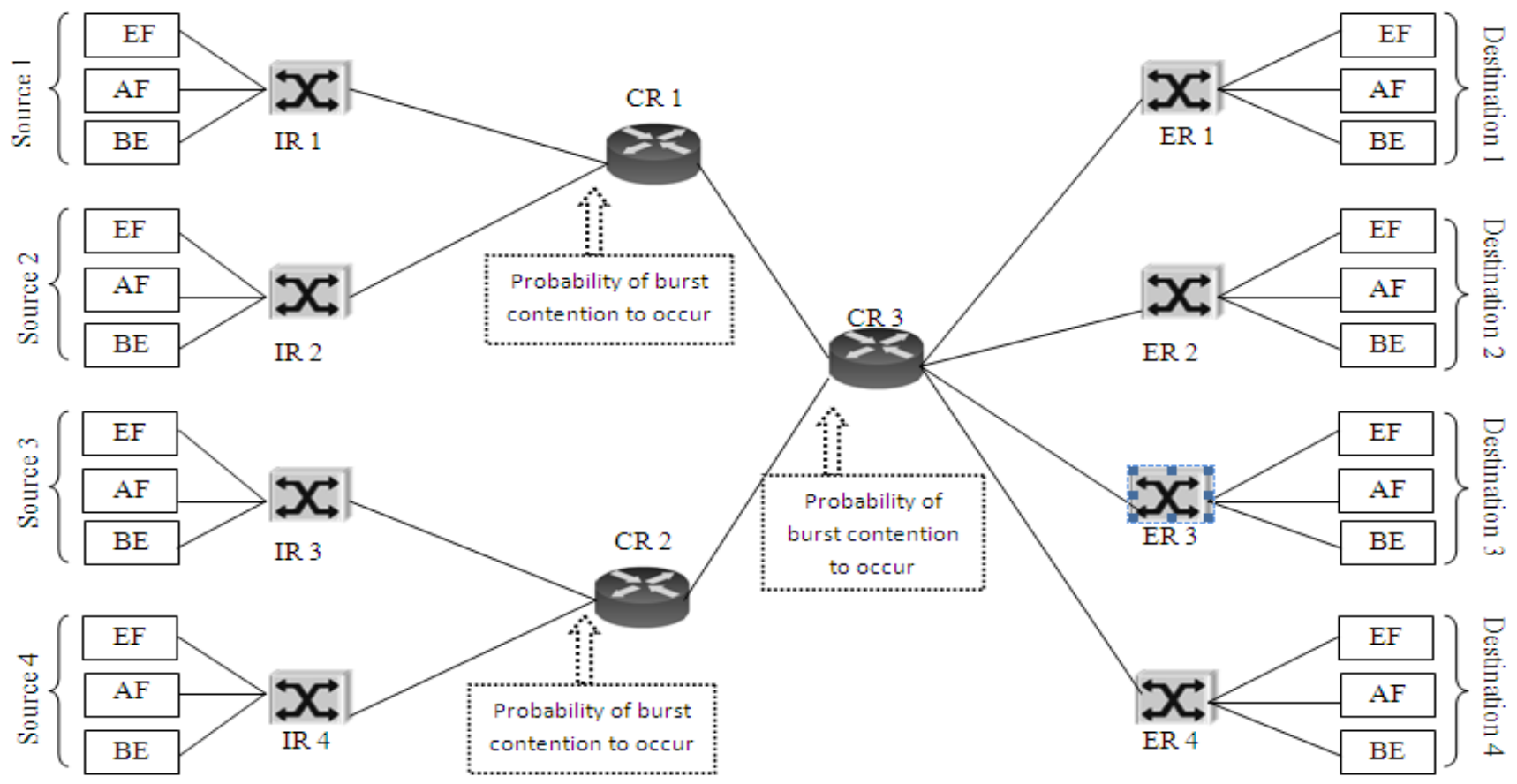

Fig. 3. Propose OBS network model.

\section{SimULATION ENVIRONMENT}

The DiffServ-aware OBS network model used in this work is shown in Fig. 3 is assumed able to cater for three classes of multimedia traffic, i.e. real time constant bit rate (CBR) traffic or Expedited Forwarding (EF) traffic, realtime variable bit rate (rtVBR) traffic or Assured Forwarding (AF) traffic and non-real time variable bit rate (nrtVBR) traffic or Best Effort (BE) traffic. The EF traffic is associated with the uniform traffic pattern and AF traffic is associated with the Pareto traffic pattern. Whereas the BE traffic corresponds to the traffic of the traditional Internet that follows Poisson traffic pattern.

The link rate between the sources to the ingress node is set to be at $100 \mathrm{Mbps}$ as it will represent the common LAN network in the real environment whereas $25 \mathrm{Mbps}$ of link rate is used between the ingress nodes to the core node. Since this is an optical transmission, the links between the cores are set to be reasonably high at $10 \mathrm{Gbps}$ as mostly used in studies. In this topology, it is assumed that each node has no wavelength conversion capability.

The size of the IP packets is variable in size corresponding to its application in the system. The EF traffic is set to have a fixed size of 256 bytes since it is suitable for uncompressed video or voice sources [16], whereas the AF packet size is fixed at 1000 bytes because it is suggested in [17] that packet size for video should not exceed Maximum Transfer Unit (MTU) which is 1500 bytes. Meanwhile, 1500 bytes is the size for the BE traffic class.

In this propose network, JET signaling is used whereby in this signaling method, sufficient time is allocated for the data burst to be transmitted without colliding into the BHP.
The type of service and the traffic generated together with its traffic rate and packet size is shown in Table I [18]. As for the burst assembly parameters; the timer burst assembly uses a preset time for each traffic which is illustrated in Table II [19]. EF has the lowest timer for it since its QoS requirement stringently avoiding delay.

TABLE I: TRAFFIC DESCRIPTION (PACKET)

\begin{tabular}{|c|c|c|}
\hline Type of Service & $\begin{array}{c}\text { Traffic Rate } \\
\text { (kbps) }\end{array}$ & $\begin{array}{c}\text { Traffic Type \& Packet } \\
\text { Size }\end{array}$ \\
\hline EF premium & 284 & CBR, 256 bytes \\
\hline AF assured & 384 & Pareto, 1000 bytes \\
\hline BE best effort & 256 & Poisson, 1500 bytes \\
\hline
\end{tabular}

TABLE II: TRAFFIC DESCRIPTION (BURST)

\begin{tabular}{|c|c|}
\hline Type of Service & Fixed Timer Burst (ms) \\
\hline EF premium & 4.8 \\
\hline AF assured & 55 \\
\hline BE best effort & 600 \\
\hline
\end{tabular}

\section{Performance Evaluation}

The performance metric used in evaluating the contention resolution techniques is the burst loss probability (BLP) according to the burst assembly technique that is fixed timer burst. In timer-based burst assembly approaches, a burst is created and sent into the optical network at periodic time intervals [20]. A timer-based scheme is used to provide uniform gaps between successive bursts from the same ingress node into the core networks. In this scheme, the length of the burst varies as the load changes.

To study the performance of the contention resolution schemes, the EF premium traffic is first gradually admitted to the OBS network from a smaller to a bigger offered traffic load, i.e. 0.1 to 0.9 , whereas the assured and best 
effort offered traffic load are fixed with 0.2 respectively. Then, the simulation work is repeated but with AF traffic load varied from 0.1 to 0.9 and the EF and BE traffic load held constant at 0.2. This is then again repeated with BE traffic class load varied from 0.1 to 0.9 and the EF and AF class load held constant at 0.2 .

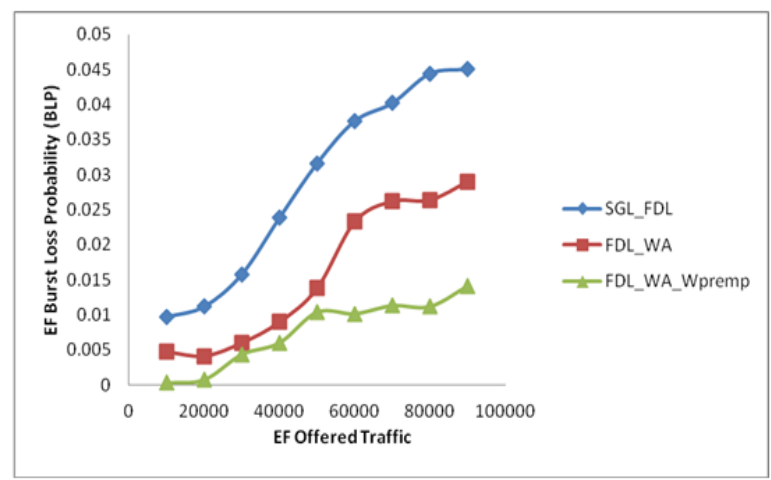

Fig. 4. EF Burst Loss Probability when EF offered traffic is increased.

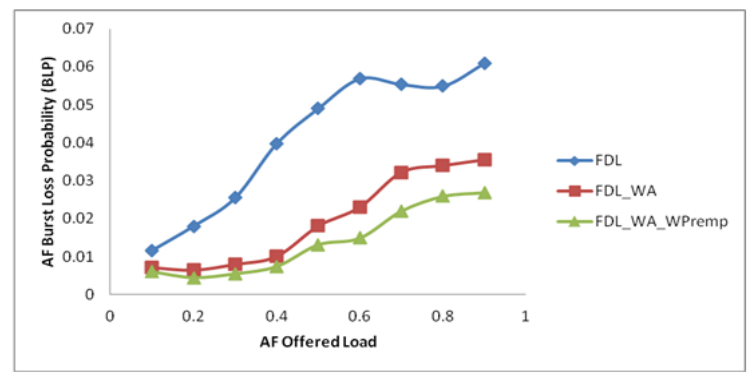

Fig. 5. AF burst Loss probability when AF offered traffic is increased.

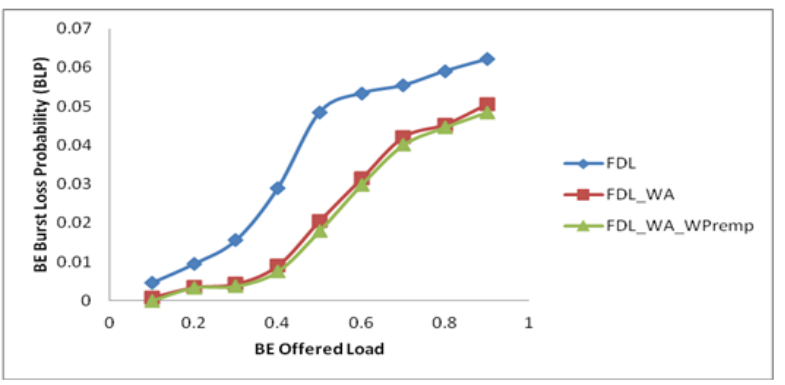

Fig. 6. BE burst loss probability when BE offered traffic is increased.

TABLE III: PERFORMANCE ACHIEVED WHEN EF OFFERED LOAD INCREASED

\begin{tabular}{|l|c|}
\hline \multicolumn{1}{|c|}{$\begin{array}{c}\text { Contention Resolution } \\
\text { Techniques }\end{array}$} & $\begin{array}{c}\text { Performance } \\
\text { Improved }\end{array}$ \\
\hline EF FDL to EF FDL_WA & $32 \%$ \\
\hline $\begin{array}{l}\text { EF FDL_WA to EF } \\
\text { FDL_WA_WPremp }\end{array}$ & $48 \%$ \\
\hline $\begin{array}{l}\text { AF FDL_WA to AF } \\
\text { FDL_WA_WPremp }\end{array}$ & $23 \%$ \\
\hline $\begin{array}{l}\text { BE FDL to BE FDL_WA \& BE } \\
\text { FDL_WA_WPremp }\end{array}$ & $33 \%$ \\
\hline
\end{tabular}

From Table III above, the burst blocking probability for all traffic class, i.e. $\mathrm{EF}, \mathrm{AF}$ and $\mathrm{BE}$ are significantly improved by $23 \%$ to $48 \%$ when using the combination of FDL, wavelength assignment and preemption compared to when there is only FDL and wavelength assignment in the case where EF traffic load is increased and AF and BE load are constant and this is also shown in Fig. 4.

In the case where AF traffic load increases and EF and $\mathrm{BE}$ traffic load held constant, the improvement achieved is moderately for the combination of three contention resolution techniques compared to with only two being implemented as illustrated in Table IV and is illustrated as in Fig. 5. Significant improvement of $25 \%$ is seen in $\mathrm{AF}$ FDL_WA to AF FDL_WA_WPremp. However, the improvement in AF FDL_WA_WPremp causes a slight increase of $8 \%$ in BE FDL_WA_WPremp when compared to BE FDL_WA.

When the BE traffic load increases and EF and AF traffic held constant, not much differences that is approximately $11 \%$ is observed between EF FDL_WA and EF FDL_WA_WPremp. This percentage obtained is supported by the graphs shown in Fig. 6. The same goes for the AF FDL_WA compared to AF FDL_WA_WPremp with an improvement of only $8 \%$. A considerable improvement is obtained from the BE traffic of about $33 \%$ when wavelength assignment scheme is added to its contention resolution techniques. However, the discrepancy of achievement when preemption is applied is almost no effect to the BE traffic class due to its nature of not being able to preempt and source (see Table V).

TABLE IV: PERFORMANCE ACHIEVED WHEN AF OFFERED LOAD INCREASED

\begin{tabular}{|l|c|}
\hline Contention Resolution Techniques & $\begin{array}{c}\text { Performance } \\
\text { Improved }\end{array}$ \\
\hline EF FDL to EF FDL_WA & $35 \%$ \\
\hline $\begin{array}{l}\text { EF FDL_WA to EF } \\
\text { FDL_WA_WPremp }\end{array}$ & $20 \%$ \\
\hline AF FDL to AF FDL_WA & $38 \%$ \\
\hline $\begin{array}{l}\text { AF FDL_WA to AF } \\
\text { FDL_WA_WPremp }\end{array}$ & $25 \%$ \\
\hline $\begin{array}{l}\text { BE FDL to BE FDL_WA \& } \\
\text { FDL_WA_WPremp }\end{array}$ & $35 \%$ \\
\hline
\end{tabular}

TABLE V: PERFORMANCE ACHIEVED WHEN BE OFFERED LOAD INCREASED

\begin{tabular}{|l|c|}
\hline Contention Resolution Techniques & $\begin{array}{c}\text { Performance } \\
\text { Improved }\end{array}$ \\
\hline EF FDL to EF FDL_WA & $31 \%$ \\
\hline $\begin{array}{l}\text { EF FDL_WA to EF } \\
\text { FDL_WA_WPremp }\end{array}$ & $11 \%$ \\
\hline AF FDL to AF FDL_WA & $30 \%$ \\
\hline AF FDL_WA to AF & $8 \%$ \\
FDL_WA_WPremp & $33 \%$ \\
\hline $\begin{array}{l}\text { BE FDL to BE FDL_WA \& } \\
\text { FDL_WA_WPremp }\end{array}$ & \\
\hline
\end{tabular}

\section{CONCLUSIONS}

These findings prove that the combination of several contention resolution techniques such as the FDL_WA_WPremp can successfully improve a low burst loss probability for applications with QoS restrictions like the EF and AF traffic class. The preemption efficiency in the contention resolution scheme improves the burst loss probability by regulating the preemption based on amount of traffic and bandwidth utilization available. This is due to the fact the preempting situations are a form of bandwidth sharing in order to avoid contention to happened between the traffic classes. Once a higher priority traffic class improves significantly, it may cause some degradation in lower priority traffic class.

\section{REFERENCES}

[1] M. Yoo and C. Qiao, "Just-enough-time (JET): A high speed Protocol for bursty traffic in optical networks," Digest of the IEEE/LEOS Summer Topical Meeting, vol. 32, no. 3, 1997. 
[2] K. Dolzer and C. Gauger, "On burst assembly in optical burst switching networks-A performance evaluation of just-enough-time," International Teletraffic Congress, vol. 4, 2001.

[3] J. M. Pitts and J. A. Schormans, Introduction to IP and ATM Design and Performance, $2^{\text {nd }}$ edition, New York: J. Wiley, 2000.

[4] J. Postel, User Datagram Protocol, RFC 768, IETF, August 1980.

[5] H. Schulzrinne, S. Casner, R. Federick, and V. Jacobson, A Transport Protocol for Real-Time Applications, 1996.

[6] M. Yoo, C. Qiao, and S. Dixit, "QoS performance of optical burst switching in IP-over-WDM networks," Journal Selected Areas Communications, vol. 18, no. 10, 2000, pp. 2062-2071.

[7] M. Yoo and C. Qiao, "A new optical burst switching protocol for supporting quality-of-service," Telecommunication Systems, vol. 34, pp. 181-194, 1998.

[8] C. Qiao, "Labeled optical burst switching for IP-over-WDM integration," Communications Magazine, vol. 38, no. 9, 2000, pp. 104-114.

[9] Z. Roseberg, A. Zalesky, H. L. Vu, and M. Zukerman, "Analysis of OBS networks with limited wavelength conversion," Transaction on Networking, vol. 14, no. 5, pp. 1118-1127, 2006.

[10] V. Vokkarane, J. P. Jue, and S. Sitaraman, "Burst segmentation: An approach for reducing packet loss in optical burst switched networks," in Proc. the IEEE International Conference on Communications (ICC), vol. 5, 2002, pp. 2673-2677.

[11] A. Detti, V. Eramo, and M. Listanti, "Optical burst switching with burst drop: An easy OBS improvement," in Proc. the IEEE International Conference on Communication (ICC), vol. 5, 2002, pp. 2687-2691..

[12] A. Detti, V. Eramo, and M. Listanti, "Performance evaluation of a new technique for IP support in a WDM optical network: Optical composite burst switching (OCBS)," Journal Lightwave Technology, vol. 20, no. 2, pp. 154-165, 2002.

[13] Y. G. Lee, N. U. Kim, T. W. Um, and M. H. Kang, "Decision of the fiber delay line length in optical burst switching networks," in Proc. the. International Conference on Advanced Communication Technology, ICACT, vol. 2, 2005, pp. 1049-1051.

[14] Y. Khlifi, A. Lazzez, S. E. F. Guemara, and N. Boudriga, "Optical packet and burst switching node architecture: Modeling and performance analysis," Telecommunications, vol. 2, pp. 507-514, 2005.

[15] M. H. S. Morsy, M. Y. S. Sowailem, and H. M. H. Shalaby, "Upper and lower bounds of burst loss probability for a core node in an optical burst switched network with pareto distributed arrivals," International Conference on Telecommunications, pp. 523-527, 2010.

[16] S. Keshav, C. R. Kalmanek, and H. Kanakia, "Rate controlled Server for very high speed networks," Global Telecommunications Conference and Exhibition Communications Connecting the Future (GLOBECOM), vol. 1, pp. 12-20, 1990.

[17] N. M. Din and N. Fisal, "Modeling of IP-GMPLS multimedia traffic and network for admission control using NS-2," in Proc. the International Conference on Modeling and Simulation, 2008, pp. 678-683.

[18] J. Baliga, E. W. M Wong, and M. Zukerman, "Analysis of bufferless OBS/OPS networks with multiple deflections," IEEE Communication Letters, vol. 13, no. 12, pp. 974-976, 2009.

[19] J. P. Jue, W. H. Yang, C. Y. Kim, and Q. Zhang, "Optical packet and burst switched networks: A review," IET Journal, vol. 3. no. 3, pp. 334-352, 2009.

[20] K Long, R. S. Tucker, and C. Wang, "A new framework and burst assembly for IP DiffServ over optical burst switching networks," The Architectures and Key Issues for the Optical Internet, pp. 3159-3164, 2003.

Suriani Mohd Sam is a member of IACSIT. She received her bachelor degree in electrical engineering (B.Eng) from University of Technology Malaysia in 1999 and She received her master degree in electrical engineering (M.Eng) in 2003 and She received her doctor of philosophy (Ph.D) in electrical engineering in 2013. Her main research interests include QoS for the optical burst switching (OBS) network.

She has been in the research field of networking particularly in qualityof-service (Qos) for several years. These includes in the field of data communication, wireless communication and telecommunication. She has published a number of international proceedings and journals in the field of QoS for several types of communication networking strategies. She is currently working as a lecturer in the Department of Electrical Engineering, Universiti Teknologi Malaysia and she currently is the head Department of the Franchise Programmer. In addition, she is also the head of Communication Engineering Programmerin the Electrical Engineering Department.

Dr. Suriani Mohd Sam is a member of the Academic Advisory Board of Kolej Yayasan Pelajaran Johor. Besides that, Dr. Suriani is also a member of the Proposal Evaluation Panel of the Research University Grant (GUP). 